\title{
Produção de leite e desempenho pós parto de vacas Hereford em distintas condições reprodutivas criadas extensivamente ${ }^{1}$
}

\author{
Milk production and postpartum performance of Hereford cows in different reproduction \\ condictions in a range breeding system
}

\author{
Marcelo Alves Pimentel ${ }^{2}$ José Carlos Ferrugem Moraes $^{3}$ Carlos Miguel Jaume $^{3}$ \\ Jaqueline Schneider Lemes ${ }^{4}$ Cássio Cassal Brauner ${ }^{4}$
}

\section{RESUMO}

A produção de leite e o desempenho pós parto de 52 vacas multíparas Hereford em condição corporal média (3 em escala de 1 a 5), gestantes (45) e não gestantes (24), em condições extensivas, foram estudados, em 69 lactações, durante dois anos (2000 e 2001). A estimativa da produção de leite foi realizada em seis intervalos ( 3 de 21 de 3 de 42 d), do nascimento à desmama (189d), por meio da técnica da pesagem do terneiro antes e após a mamada. Os fatores fixos foram ano, prenhez, sexo do terneiro e raça do touro (Hereford e Nelore). A produção de leite diária, do pico de lactação, total e a persistência da lactação, não foram influenciadas pelos fatores estudados $(P>0,05)$. O peso ao parto foi maior $(P<0,05)$ em $2001,(417,49$ $\pm 6,94 \mathrm{~kg})$, que em 2000 (353, $97 \pm 5,57 \mathrm{~kg})$, e não teve efeito $(P>0,05)$ dos demais fatores avaliados. O peso vivo na concepção (99 d média), e à desmama, foi maior nas vacas gestantes (432,10 $\pm 5,78$ e 433,30 $\pm 6,36 \mathrm{~kg}$ ) que nas não gestantes 399,37 \pm 7,92 e $393,46 \pm 8,70 \mathrm{~kg})$, respectivamente. O sexo dos terneiros teve efeito significativo $(P<0,05)$ sobre peso ao nascer $(41,75 \pm 1,21$ e 37,6 $\pm 1,13 \mathrm{~kg}$, para machos e fêmeas, respectivamente). No peso à desmama, todos os fatores demonstraram efeito $(P<0,05)$ e os terneiros das vacas gestantes $(194,22 \pm 3,41 \mathrm{~kg})$ foram mais pesados que das vazias $(177,43 \pm 4,67 \mathrm{~kg})$. Em vacas de corte com produção de leite e condição corporal similar, do parto à desmama, o melhor desempenho das gestantes, em peso vivo $e$ peso do terneiro à desmama demonstra que, provavelmente, uma melhor adaptação ao sistema tenha sido responsável pela fertilidade pós-parto.

Palavras-chave: produção de leite, desempenho pós-parto, bovinos de corte.

\begin{abstract}
Milk production and pospartum performance from 52 multiparous Hereford cows, with the same body condition score (3 in a 1 to 5 scale), pregnant (45) or not pregnant (24), was studied in 69 lactations during two years, 2000 (42) and 2001 (27) under range conditions. Estimates of milk yield were obtained during six intervals (three 21d and three 42d), by the weigh-suckle-weigh technique, from birth to weaning (189d). Effects studied were year, pregnancy, calf sex and sire breed. None of the effects studied affected $(P>0.05)$ milk production parameters: daily milk production, total milk yield, lactation peak and persistency. Body weight at calving was greater $(P<0.05)$ in 2001 $(417.49 \pm 6.94 \mathrm{~kg})$ that in $2000(353.97 \pm 5.57 \mathrm{~kg})$ and was not affected by the other effects. Body weight at conception (99d; $432.10 \pm 5.78$ and $399,37 \pm 7.92 \mathrm{~kg}$ ) and at weaning $(189 \mathrm{~d} ; 433.30 \pm 6.36$ and $393.46 \pm 8.70 \mathrm{~kg})$, were greater $(P<0.05)$ in pregnant cows than in non-pregnant animals, respectively. Sex of calf had a significant effect $(P<0.05)$ on calf birth weight $(41.75 \pm 1.21 \mathrm{~kg}$ and $37.6 \pm 1.13 \mathrm{~kg}$ for males and females, respectively). All studied effects affected significantly calf weaning weight $(P<0.05)$ and pregnant cows had heavier calves at weaning $(194.22 \pm 3.41 \mathrm{~kg})$ than non-pregnant cows $(177.43 \pm 4.67 \mathrm{~kg})$. The greatest performance of pregnant beef cows with similar milk yield and body condition score from calving to weaning can be explained by the superior adaptation characteristics of these cows.
\end{abstract}

Key words: milk production, postpartum performance, beef cattle.

${ }^{1}$ Parte da tese de doutorado do primeiro autor apresentada ao Programa de Pós-graduação em Zootecnia, Faculdade de Agronomia Eliseu Maciel (FAEM), Universidade Federal de Pelotas (UFPel), como parte das exigências para obtenção do título de Doutor em Ciências. ${ }^{2}$ Professor Adjunto do Departamento de Zootecnia, FAEM, UFPEL, CP 354, CEP 96010-900, Pelotas, RS. E-mail: map@ufpel.tche.br Autor para correspondência.

${ }^{3}$ Pesquisador da Embrapa, Pecuária Sul, Doutor

${ }^{4}$ Acadêmico da Veterinária, Estágiário do Departamento de Zootecnia, FAEM, UFPEL. 


\section{INTRODUÇÃO}

A reprodução é o fator que mais limita a produtividade em bovinos de corte e as maiores perdas decorrem de falhas reprodutivas (SHORT et al., 1990, BUCHANAN et al., 2000). A determinação de um período fixo de acasalamento para vacas de corte torna a fertilidade pós-parto o fator chave quando o objetivo é a obtenção de um terneiro por ano (BARTLE et al., 1984). Entre fatores que afetam a fertilidade pós-parto, destacam-se, o fotoperíodo, o bioestímulo (feromônios), o estresse e fundamentalmente, a nutrição e a amamentação (PIMENTEL et al., 1979; SHORT et al., 1990; JAUME, 2000). A hipótese de prioridade da síntese do leite sobre a atividade reprodutiva, quanto à partição de nutrientes tem motivado pesquisas que relacionam a produção de leite com a fertilidade pós-parto (SHORT et al., 1990; HUNTER \& D'OCCHIO, 1995; BAUMAN, 2000). O aspecto mais estudado é o tempo de retorno à atividade reprodutiva ou de anestro pós-parto, ou ainda intervalo pós-parto (IPP). Aumentos de 1,4, 3,3, e 5,1 dias de acréscimo no IPP, para cada litro de leite produzido, foram observados nos trabalhos de BOGGS et al. (1980), BARTLE et al. (1984) e ALENCAR (1987b). Estas observações sugerem menor eficiência reprodutiva para vacas que produzem mais leite, em raças de corte européias e zebuínas (ALENCAR et al., 1993; LOPES et al., 2000). Por outro lado, a inexistência de correlação significativa entre produção de leite e IPP, diferença na atividade luteal entre vacas de alta e baixa habilidade materna, assim como efeito do estímulo da mamada sobre a fertilidade de vacas com diferentes produções de leite, sugere a independência entre produção de leite e fertilidade das vacas (DAY et al., 1987; BEAL et al., 1990; BUCHANAN et al., 2000).

A relação entre condição corporal ao parto e produção de leite em vacas de corte ainda é pouco estudada (LALMAN et al., 2000). A avaliação da condição corporal é uma metodologia útil para inferir sobre condição nutricional dos bovinos, sua atividade ovariana e futuro desempenho reprodutivo (MORAES \& JAUME, 2000; JAUME \& MORAES, 1996; JAUME \& MORAES 2002). Neste contexto, mudanças de peso pré e pós-parto tem pouco efeito em vacas em boa condição corporal, entretanto, para vacas magras o ganho de peso pós-parto é importante (DUNN \& KALTENBACH, 1980). Nesse sentido, foi demonstrado que a melhoria da condição corporal da vaca quatro meses após o parto é associada à redução da produção de leite (JEFFERY et al., 1971; BOGGS et al., 1980). De um modo geral, tem sido relatada uma correlação alta e significativa entre redução na condição corporal ao parto e maior produção de leite diária nos primeiros 90 dias de lactação, o que pode ser indicativo de que vacas com maior habilidade em manter a condição corporal em períodos de restrição alimentar pré-parto têm menor potencial para produzir leite (LALMAN et al., 2000). Assim, a maioria dos estudos concluem que a variação da condição corporal pós-parto e conseqüentes diferenças no IPP poderiam ser explicadas pela produção de leite. Com base na literatura revisada, observam-se divergências quanto aos efeitos da produção de leite, condição corporal pré e pós-parto e desempenho reprodutivo, porém a maior parte dos estudos focaram na recuperação das vacas durante períodos limitados. O presente estudo teve como objetivo verificar o desempenho produtivo de vacas de corte prenhes ou não, em uma mesma condição corporal, média (3 em escala de 1 a 5), do parto à desmama.

\section{MATERIAL E MÉTODOS}

Foram avaliadas 69 lactações de 52 vacas da raça Hereford, durante dois anos, sendo 42 em 2000 e 27 em 2001 (17 das quais faziam parte do lote de 2000), paridas nos meses de setembro e outubro. As vacas incluídas neste estudo tinham idade entre 5 a 7 anos e se mantiveram em condição corporal (CC) 3 numa escala de 1 a 5 (BUSKIRK et al., 1992; JAUME \& MORAES, 2002), do parto à desmama. No primeiro ano, os terneiros eram filhos de touro Nelore, no segundo 20 de Hereford e sete de Nelore. Os animais foram mantidos em campo natural, em uma lotação de $0,7 \mathrm{UA} \mathrm{ha}{ }^{-1}(\mathrm{UA}=$ unidade animal $=450 \mathrm{~kg}$ de peso vivo).

O trabalho foi conduzido em uma fazenda particular no município Aceguá, localizado na região fisiográfica denominada de Campanha, Estado do Rio Grande do Sul, entre os paralelos $30^{\circ} 30^{\prime}$ e $31^{\circ} 56^{\prime}$ Sul e os meridianos de $55^{\circ} 30^{\prime}$ e $54^{\circ} 30^{\prime}$ a Oeste de Greenwich. A região apresenta clima mesotérmico, tipo subtropical da classe Cfa 1, de acordo com a classificação de Köppen, com chuvas distribuídas uniformemente durante o ano, média é de 1350mm, variando entre 1080 e $1620 \mathrm{~mm}$, 34\% no inverno, 25\% na primavera, $16 \%$ no verão e $25 \%$ no outono. Entre os meses de novembro a março, podem ocorrer períodos secos (MACEDO, 1984). Durante os períodos experimentais (setembro a abril), a precipitação pluviométrica foi de 1384mm em 2000/ 2001, e de $1661 \mathrm{~mm}$ em 2001/2002. A temperatura média anual é de $17,6^{\circ} \mathrm{C}$, sendo janeiro o mês mais quente, com média de $24^{\circ} \mathrm{C}$, e junho o mais frio, com 
$12,5^{\circ} \mathrm{C}$. Geadas podem ocorrer entre abril e novembro, com maior incidência de junho a agosto. A umidade relativa do ar oscila entre $75 \%$ e $85 \%$. O relevo da região é suavemente ondulado, apresentando longos declives de 2 a 8 . A altitude varia entre 80 e 180 metros acima do nível do mar. A fertilidade do solo é moderada, com baixos teores de fósforo. A cobertura natural do solo é superior a $80 \%$, formada por vegetação nativa, com variações qualitativas durante o ano. Na primavera e verão, a vegetação nativa é baixa e densa, de boa qualidade e no inverno (junho a agosto), não apresentam crescimento e crestam em razão das geadas. A população de plantas predominante é de gramíneas de ciclo estival, sendo formada principalmente pela "grama forquilha" (Paspalum notatum) e "grama tapete" (Axonopus affinis). As gramíneas de ciclo hibernal de maior ocorrência são as "flexilhas” (Stipa spp), e o “azevém" (Lolium multiflorum spp). A população de leguminosas de ciclo hibernal é na sua maior parte composta por trevos (Tripholium spp).

A produção de leite foi estimada pelo método de pesar o terneiro antes e após a mamada (BARTLE et al., 1984; BEAL et al., 1990). Os terneiros foram separados das vacas no dia anterior ao da pesagem (6h). Ao final da tarde (18h), foram colocados a mamar, para esgotamento do úbere e separados por $12 \mathrm{~h}$. Na manhã seguinte (6h), foram pesados em jejum e colocados para mamar entre 20 a $30 \mathrm{~min}$, posteriormente pesados e a diferença entre os pesos considerada a produção de leite de $12 \mathrm{~h}$, que multiplicada por dois foi utilizada como a estimativa para 24h. Foram realizadas seis medidas, as três primeiras em intervalos de 21 as outras em intervalos de 42 dias, totalizando um período de 189 dias. Para as pesagens foi utilizada balança eletrônica ${ }^{1}$, com capacidade máxima de $1500 \mathrm{~kg}$ e precisão de $100 \mathrm{~g}$. O peso vivo e a condição corporal (CC) das vacas foram controlados do parto ao desmame.

A produção de leite, nos períodos foi estimada pela fórmula proposta por ALENCAR et al. (1996): $P L_{n}=\left[\left(L_{n}+L_{n-1}\right) / 2\right] \times 21$, onde $P L_{n}$ é a produção de leite do período de 21 dias, $L_{n}$ é a produção de leite estimada no dia, ou seja a produção observada no dia da pesagem multiplicada por dois e $\mathrm{n}=21 ; 42 ; . . ., 189$. A produção de leite total (PLT) de cada vaca foi estimada somando-se todas as PLn (PLT $=$ å $\mathrm{PL}_{n}$ ). A persistência da lactação (PER) foi calculada por meio da relação entre a diferença da última produção diária (PL9) e da mais alta produção verificada (PL2) pelo período em dias entre elas: PL9 - PL2/147 dias. Esta variável é expressa em g dia-1, e significa o quanto se reduz a produção média de leite por dia, a partir do pico de lactação (JENKINS et al., 2000).

As vacas foram submetidas à sincronização de cio entre 30 a 100 dias após o parto. Foi utilizado o método descrito por SOUZA \& MORAES (1998), empregando pessários (esponjas intra-vaginais impregnadas com 250mg de acetato de medroxiprogesterona) por sete dias, e aplicação de $5 \mathrm{mg}$ de benzoato de estradiol (intramuscular) no momento da colocação. Após a retirada dos pessários, os terneiros foram separados das vacas por quatro dias, e realizada inseminação artificial (IA) pelo método convencional, com observação de cio. Em seqüência, as vacas foram acasaladas em monta natural por 50 dias. O diagnóstico de gestação foi realizado 60 dias após o término do acasalamento e as vacas controladas até o parto para verificar a data da concepção.

Para análise dos dados, foram considerados fatores fixos, ano (A), prenhez (P), sexo do terneiro $(S)$, e raça do touro $(R)$, as interações entre efeito de ano e prenhez (AP), ano e sexo do terneiro (AS) e prenhez e sexo do terneiro (PS). Como variáveis resposta, foram analisadas a produção total de leite (PLT), a produção de leite nas medidas diárias (PL), a persistência da lactação (PER), o peso da vaca ao parto (PPA), o peso da vaca à concepção (PCO) (nesta variável, para efeito de comparação, o peso das vacas vazias foi considerado na média da data de concepção das gestantes), o peso da vaca à desmama (PVD), o peso do terneiro ao nascer (PTN), o peso do terneiro nas pesagens (PT) e ao desmame, corrigido para 189 dias (PTD). Os dados foram submetidos à análise da variância (ANOVA - GLM), no programa NCSS 6.0 (1996), em função da disponibilidade de informações nos distintos anos.

Para análise do intervalo (dias) parto concepção, ou pós-parto (IPP), foram utilizadas apenas as informações das vacas gestantes e o modelo foi o seguinte:

$\mathrm{Y}_{\mathrm{ijk}}=\mathrm{m}+\mathrm{A}_{\mathrm{i}}+\mathrm{S}_{\mathrm{j}}+\mathrm{R}_{\mathrm{k}}+\mathrm{A} \mathrm{S}_{\mathrm{ij}}+\mathrm{e}_{\mathrm{ijk}}$, onde: $\mathrm{Y}_{\mathrm{ijk}}=$ uma observação de IPP, $\mathrm{m}=$ média geral, $\mathrm{A}_{\mathrm{i}}=$ efeito de ano ( $\mathrm{i}=2000,2001), \mathrm{S}_{\mathrm{j}}=$ efeito do sexo do terneiro ( $j=$ macho, fêmea), $R_{k}=$ efeito da raça do touro ( $\mathrm{k}=$ Hereford, Nelore), $\mathrm{AS}_{\mathrm{ij}}=$ interação entre efeito de ano sexo do terneiro, $\mathrm{e}_{\mathrm{ijk} .}=$ erro experimental.

\section{RESULTADOS E DISCUSSÃO}

As médias ajustadas de produção de leite diária (PLD), pico de lactação (42d), produção de leite total (PLT) e persistência da lactação, estão apresentadas na tabela 1 . Merece destaque o fato que as vacas gestantes produziram $102,76 \mathrm{~kg}$ a mais na

Ciência Rural, v.35, n.1, jan-fev, 2005. 
Tabela 1 - Médias ajustadas e erro padrão da produção de leite média diária (PLD), pico de lactação, produção de leite total (PLT) e persistência da lactação (PER), nos fatores ano, prenhez ( $\mathrm{P}=$ prenhe, $\mathrm{V}=$ vazia), sexo do terneiro $(\mathrm{M}=\mathrm{macho}, \mathrm{F}=$ fêmea) e raça do touro $(\mathrm{H}=$ Hereford, $\mathrm{N}=$ Nelore)

\begin{tabular}{|c|c|c|c|c|c|}
\hline \multicolumn{2}{|l|}{ Fatores } & PLD (kg) & Pico de Lactação (kg) & PLT (kg) & PER (g/dia) \\
\hline \multicolumn{6}{|l|}{ Ano } \\
\hline 2000 & 42 & $5,41 \pm 0,17$ & $6,98 \pm 0,34$ & $1028,24 \pm 34,00$ & $-35,62 \pm 2,68$ \\
\hline 2001 & 27 & $4,97 \pm 0,21$ & $7,50+0,42$ & $1004,98 \pm 42,40$ & $-22,97 \pm 3,34$ \\
\hline \multicolumn{6}{|c|}{ Prenhez } \\
\hline $\mathrm{P}$ & 45 & $5,37 \pm 0,17$ & $7,38 \pm 0,33$ & $1067,99 \pm 32,85$ & $-28,41 \pm 2,59$ \\
\hline $\mathrm{V}$ & 24 & $5,01 \pm 0,23$ & $7,10 \pm 0,45$ & $965,23 \pm 44,98$ & $-30,18 \pm 3,54$ \\
\hline \multicolumn{6}{|c|}{ SexoTern } \\
\hline $\mathrm{F}$ & 37 & $4,93 \pm 0,18$ & $6,82 \pm 0,36$ & $969,48 \pm 36,22$ & $-27,33 \pm 2,85$ \\
\hline M & 32 & $5,46 \pm 0,20$ & $7,66 \pm 0,39$ & $1063,74 \pm 38,95$ & $-31,26 \pm 3,07$ \\
\hline \multicolumn{6}{|c|}{ RaçaTouro } \\
\hline $\mathrm{H}$ & 20 & $4,96 \pm 0,25$ & $6,56 \pm 0,49$ & $994,58 \pm 49,27$ & $-31,54 \pm 3,88$ \\
\hline $\mathrm{N}$ & 49 & $5,42 \pm 0,16$ & $7,92 \pm 0,32$ & $1038,64 \pm 31,48$ & $-27,05 \pm 2,48$ \\
\hline Geral & 69 & 5,19 & 7,24 & 1016,609 & $-29,29$ \\
\hline
\end{tabular}

PLT (189d) que as vazias, porém a diferença não foi significativa $(\mathrm{P}>0,05)$. Nenhum dos outros fatores estudados teve efeito significativo $(\mathrm{P}>0,05)$ sobre as variáveis de produção de leite. Estes resultados sugerem que possivelmente outros fatores tenham influenciado o desempenho reprodutivo das vacas, contrariando os trabalhos de BOGGS et al. (1980), BARTLE et al. (1984), ALENCAR (1987b), ALENCAR et al. (1993) e LALMAN et al. (2000), que incluem a produção de leite como um fator decisivo no sucesso reprodutivo. No presente estudo, todas as vacas mantiveram-se em CC média (3) durante a lactação e sem diferença nas características de produção de leite. Os resultados estão mais de acordo com os de DAY et al. (1987), BEAL et al. (1990), SPITZER et al. (1995) e BUCHANAN et al. (2000), que não relacionam a maior produção de leite a insucesso reprodutivo e menores reservas corporais. Desta forma, a concepção deve ser explicada por outros fatores que não a produção de leite, diferenças de CC, ou ainda a produção de leite influenciando negativamente a CC e o IPP.

As médias ajustadas de peso vivo das vacas, ao parto, à concepção (103d) e à desmama, estão apresentadas na tabela 2. No ano de 2001, as vacas pesaram $63,52 \mathrm{~kg}$ a mais no parto $(\mathrm{P}<0,05)$ que as de 2000. Os demais fatores não apresentaram efeito significativo $(\mathrm{P}>0,05)$ sobre o peso ao parto. Muitos estudos relacionam a condição nutricional ao parto à produção de leite e à fertilidade pós-parto (SPITZER et al., 1995; LALMAN et al., 2000; PIMENTEL et al., 2001). Entretanto, neste trabalho, com animais na mesma CC, sem diferença na produção de leite e peso ao parto as vacas gestantes foram mais pesadas no momento da concepção, contrariando os resultados de JEFFERY et al. (1971) de que vacas que ganham mais peso durante a lactação produzem menos leite. A explicação para essa diferença pode ser que o melhor desempenho das vacas gestantes, expresso por $32,73 \mathrm{~kg}$ a mais, embora insuficiente para promover o acréscimo de uma unidade de CC, numa escala de 1 a 5 , pode ter sido responsável pela resposta das vacas mais adaptadas, que mesmo produzindo quantidade de leite semelhante estavam aptas para conceber. $\mathrm{O}$ peso das vacas à desmama também foi maior $(\mathrm{P}<0,05)$ nas vacas gestantes, com uma diferença de 39,84kg. Indicando que provavelmente a partir da concepção o efeito anabolizante da gestação tenha influenciado o desempenho das vacas expresso em peso corpóreo.

Ainda na tabela 2, são apresentadas as médias ajustadas de peso vivo ao nascer, peso à desmama ajustado para 189 dias e ganho de peso médio diário (GMD) dos terneiros. O sexo dos terneiros foi o único fator com efeito significativo $(\mathrm{P}<0,05)$ sobre o peso ao nascer. Esse fator tem sido descrito por diversos autores e invariavelmente os machos são mais pesados que as fêmeas (CARDELLINO \& CASTRO, 1987; CARDOSO et al., 2001). No peso ajustado à desmama assim como no GMD, todos os fatores tiveram efeito significativo $(\mathrm{P}<0,05)$, sendo que ano e raça do touro apresentamse confundidos pois em 2000 o touro era Nelore e Hereford e Nelore em 2001. Quanto ao sexo, os machos mantiveram-se mais pesados e os terneiros de vacas gestantes pesaram 16,8 kg e 0,103kg de GMD a mais $(P<0,05)$. Essa diferença não pode ser 
Tabela 2 - Médias ajustadas e erro padrão do peso vivo (kg) das vacas, ao parto (PPA), à concepção (PCO) e à desmama (PVD), e dos terneiros ao nascer (PTN), à desmama (PTD) e ganho médio diário (GMD) nos fatores ano, prenhez $(\mathrm{P}=\mathrm{prenha,} \mathrm{V}=\mathrm{vazia})$, sexo ( $\mathrm{M}=$ macho, $\mathrm{F}=$ fêmea) do terneiro e raça do touro $(\mathrm{H}=$ Hereford, $\mathrm{N}=$ Nelore)

\begin{tabular}{|c|c|c|c|c|c|c|c|}
\hline \multirow{2}{*}{ Fatores } & \multirow{2}{*}{$\mathrm{n}$} & \multirow{2}{*}{ PPA } & \multirow{2}{*}{ PCO } & \multicolumn{3}{|l|}{ Peso vivo (kg) } & \multirow[b]{2}{*}{ GMD (kg) } \\
\hline & & & & PVD & PTN & PTD & \\
\hline \multicolumn{8}{|l|}{ Ano } \\
\hline 2000 & 42 & $353,97 \pm 5,57^{\mathrm{a}}$ & $408,86 \pm 5,99$ & $406,59 \pm 6,58$ & $38,39 \pm 1,06$ & $166,21 \pm 3,53^{a}$ & $0,676 \pm 0,017^{\mathrm{a}}$ \\
\hline 2001 & 27 & $417,49 \pm 6,94^{b}$ & $422,61 \pm 7,47$ & $420,17 \pm 8,21$ & $40,42 \pm 1,32$ & $205,44 \pm 4,40^{b}$ & $0,873 \pm 0,021^{b}$ \\
\hline \multicolumn{8}{|l|}{ Prenhez } \\
\hline $\mathrm{P}$ & 45 & $396,98 \pm 5,38$ & $432,10 \pm 5,78^{a}$ & $433,30 \pm 6,36^{a}$ & $38,04 \pm 1,02$ & $194,22 \pm 3,41^{a}$ & $0,826 \pm 0,017^{\mathrm{a}}$ \\
\hline $\mathrm{V}$ & 24 & $374,48 \pm 7,36$ & $399,37 \pm 7,92^{\mathrm{b}}$ & $393,46 \pm 8,70^{b}$ & $40,77 \pm 1,40$ & $177,43 \pm 4,67^{b}$ & $0,723 \pm 0,023^{\mathrm{b}}$ \\
\hline \multicolumn{8}{|c|}{ SexoTern } \\
\hline $\mathrm{F}$ & 37 & $383,53 \pm 5,93$ & $412,83 \pm 6,38$ & $411,15 \pm 7,01$ & $37,06 \pm 1,13^{a}$ & $176,52 \pm 3,76^{a}$ & $0,738 \pm 0,018^{a}$ \\
\hline M & 32 & $387,92 \pm 6,38$ & $418,64 \pm 6,86$ & $415,61 \pm 7,54$ & $41,75 \pm 1,21^{b}$ & $195,13 \pm 4,04^{b}$ & $0,812 \pm 0,018^{b}$ \\
\hline \multicolumn{8}{|c|}{ RaçaTouro } \\
\hline $\mathrm{H}$ & 20 & $389,82 \pm 8,07$ & $421,66 \pm 8,68$ & $411,25 \pm 9,53$ & $37,11 \pm 1,53$ & $167,58 \pm 5,11^{a}$ & $0,690 \pm 0,025^{a}$ \\
\hline $\mathrm{N}$ & 49 & $381,63 \pm 5,15$ & $409,81 \pm 5,54$ & $415,51 \pm 6,09$ & $41,70 \pm 0,98$ & $204,08 \pm 3,27^{b}$ & $0,859 \pm 0,016^{b}$ \\
\hline Geral & 69 & 385,73 & 415,74 & 413,38 & 39,40 & 185,83 & 0,775 \\
\hline
\end{tabular}

Letras diferentes na coluna, dentro de fatores, indicam médias diferentes $(\mathrm{P}<0,05)$.

explicada, pela maior produção de leite, como autores atribuem (NEVILLE, 1962; MELTON et al., 1967; RUTLEDGE et al., 1971; BOOGS et al., 1980; BARTLE et al., 1984; ALENCAR, 1987a; ALENCAR et al., 1996; DAY et al., 1987; BUCHANAN \& STUTTS, 2001), já que vacas gestantes e vazias não apresentaram diferença $(\mathrm{P}<0,05)$ nas características de produção de leite. $\mathrm{O}$ melhor desempenho dos terneiros filhos de touro Nelore pode ser atribuído à heterose individual, que também já foi amplamente descrita (KOGER et al., 1976; CARDELLINO \& ROVIRA, 1987).

Analisando apenas as vacas gestantes, o efeito de ano sobre o IPP foi significativo $(\mathrm{P}<0,05)$, com médias ajustadas de $114 \pm 4,9$ e $84 \pm 4,6$ dias para 2000 e 2001 respectivamente, sendo a média geral 99 dias. Os demais fatores investigados e interações não demonstraram efeito sobre o IPP $(\mathrm{P}>0,05)$. BEAL et al. (1990) observaram intervalos entre parto e primeira ovulação de $66 \pm 4$ dias com uma amplitude de 9 e 143. LALMAN et al. (2000), estudando diferentes dietas energéticas em primíparas magras, verificaram IPP de 121士2,9 dias e atribuiram à maior produção de leite o aumento no IPP. Da mesma forma, ALENCAR (1987b) aponta a maior produção de leite como responsável pelo aumento no intervalo partoprimeiro serviço, com 5,1dias kg-1 leite. ALENCAR et al. (1993) observaram IPP de 161 e 108 dias em vacas Canchim e Nelore, respectivamente, e também atribuíram a redução da eficiência reprodutiva à produção de leite. No presente estudo, vacas com cria ao pé que ficaram gestantes conceberam em 99 dias após o parto, permaneceram em igual CC durante a lactação, e com produção de leite similar à das que não emprenharam. Portanto, essa grande diferença deve ser explicada por outras causas que não a produção de leite ou seu efeito sobre a CC. Possivelmente, exista o envolvimento de outros mecanismos fisiológicos relativos ao restabelecimento precoce da atividade cíclica estral pós-parto, concordando com os estudo de OLIVEIRA et al. (2002) que avaliaram vacas de alta e baixa fertilidade.

\section{CONCLUSÃO}

Em vacas de corte da raça Hereford criadas em condições extensivas, a produção de leite não deve ser um dos principais fatores limitantes da fertilidade pós-parto, considerando que apenas as vacas que ganharam mais peso após o parto, ainda que insuficiente para melhoria de sua condição corporal, foram as aptas a conceber e ainda a produzirem terneiros mais pesados à desmama. Isto deve estar relacionado ao fato de que os animais dessa raça e aptidão apresentam um platô de produção de leite ao qual as vacas podem responder com incremento de produção frente a ofertas superiores de forragem. Além disso, é plausível inferir que existem outros fatores ou interações importantes à adaptação dos animais às condições de criação que viabilizem maior eficácia reprodutiva em vacas de corte. 


\section{REFERÊNCIAS BIBLIOGRÁFICAS}

ALENCAR, M.M. Efeitos da produção de leite sobre o desenvolvimento de bezerros Canchim. Revista da Sociedade Brasileira de Zootecnia, v.16, n.1, p.01-13, 1987a.

ALENCAR, M.M. Herdabilidade e efeito da produção de leite sobre a eficiência reprodutiva de vacas da raça Canchim. Revista da Sociedade Brasileira de Zootecnia, v.16, n.2, p.163-169, $1987 b$

ALENCAR, M.M. et al. Desenvolvimento pós-desmama e eficiência reprodutiva pós-parto em gado de corte: Influência da produção de leite. Revista da Sociedade Brasileira de Zootecnia, v.22, n.6, p.1012-1018, 1993

ALENCAR, M.M. et al. Produção de leite da vaca e desenvolvimento do bezerro em gado de corte. Revista da Sociedade Brasileira de Zootecnia, v.25, n.1, p.92-101, 1996.

BARTLE, S.J. et al. Effect of energy intake on the postpartum interval in beef cows and the adequacy of the cow's milk production for calf growth. Journal Animal Science, v.58, n.5, p.1068-1074, 1984

BAUMAN, D.E. Regulation of nutrient partioning during lactation: homeostasis and homeoresis. In:__. Ruminant physiology. Digestion, metabolism, growth and reproduction (ed.) CAB International (P.B. Cronjé), 2000. p.311-328.

BEAL, W.E. et al. Techniques for estimation of milk yield in beef cows and relationships of milk yield to calf weight gain and postpartum reproduction. Journal Animal Science, v.68, p.937943, 1990.

BOGGS, D.L. et al. Effects of milk and forage intake on calf performance. Journal of Animal Science, v.51, n.3, p.550-553, 1980.

BUCHANAN, D.S. et al. Genetic variation in days to onset of post partum ovarian function for cows sired by high and low milk EPD Hereford and Angus bulls. Capturado em 11 out. 2001. Online. Disponível em: http://www.ansi.okstate.edu/ research/2000rr/01.htm

BUCHANAN, D.S.; STUTTS, K.J. Evaluation of milk production and calf performance in range beef cows sired by high and low milk expected progeny difference Angus and Hereford bulls. Capturado em 22/nov/2001. Online. Disponível em: http://www.ansi.okstate.edu/research/2001rr/01/01.htm

BUSKIRK, D.D. et al. Estimation of netenergy requeriments (NEm and NED) of lacting beef cows. Journal of Animal Science, v.70, p.3867-3876, 1992.

CARDELlinO, R.A.; ROVIRA, J. Mejoramiento genético animal. Montevideo, Uruguay : Agropecuaria Hemisferio Sur, 1987. 253p.

CARDELLINO, R.A.; CASTRO L.F.S. Efeitos ambientais e fatores de correção para peso ao nascer, peso à desmama e ganho de peso pré-desmama, em bovinos Nelore. Revista da Sociedade Brasileira de Zootecnia, v.16, n.1, p.14-27, 1987.

CARDOSO, F.F. et al. Fatores ambientais que afetam o desenvolvimento do nascimento à desmama de bezerros Angus criados no Rio Grande do Sul. Revista Brasileira de Zootecnia, v.30, n.2 p.326-335, 2001.

DAY, M.L. et al. Suckling behavior of calves with dams varying in milk production. Journal of Animal Science, v.65, p.12071212, 1987.

DUNN, T.G.; KALTENBACH, C.C. Nutrition and pospartum the interval of the ewe, sow and cow. Journal of Animal Science, v.51 (Suppl. 2), p.29-39, 1980

HUNTER, R.A.; D’OCCHIO, M.J. Partitio nutrients and return to oestrus in lactating Bos indicus cows. Australian Journal of Agricultural Research, v.46, p.749-762, 1995.

JAUME, C.M. Control hormonal y sus interacciones en vacas amamantando. In:__ Estrategias para acortar el anestro posparto en vacas de carne. Montevideo : INIA, 2000. p.25. (Serie Técnica no 108).

JAUME, C.M.; MORAES, J.C.F. Effect of body condition on ovulation induced by medroxi-progesterone and equine chorionic gonadotrophin in suckled beef cows in Southern Brazil. In: INTERNATIONAL CONGRESS ON ANIMAL REPRODUCTION, 13., 1996, Sydney. Anais... Sydney : Australian Society of Animal Reproduction, 1996. V.3, p.1914.

JAUME, C.M.; MORAES, J.C.F. Um sistema para melhorar a taxa reprodutiva em vacas de cria. Bagé : EMBRAPA, 2001. V.37, p.1-14. (Documentos).

JAUME, C.M.; MORAES, J.C.F. Importância da condição corporal na eficiência reprodutiva do rebanho de cria. Bagé: EMBRAPA, 2002. V.43, jul, p.1-30. (Documentos).

JEFFERY, H.B. et al. Factors influencing milk yield of beef cattle. Canadian Journal of Animal Science, v.51, n.3, p.551560, 1971.

JENKINS, T.G. et al. Lactation and calf weight traits of mature crossbred cows fed varying daily levels of metabolizable energy. Journal of Animal Science, v.78, p.7-14, 2000.

KOGER, M. et al. Cruzamientos en ganado vacuno de carne. Montevideo : Agropecuaria Hemisferio Sur, 1976. 559p.

LALMAN, D.L et al. Effect of dietary energy on milk production and metabolic hormones in thin, primiparous beef heifers. Journal of Animal Science, v.78, p.530-538, 2000.

LOPES, B.C. et al. Influência da produção de leite sobre a ocorrência do primeiro cio em primíparas Bos taurus indicus. In: REUNIÃO ANUAL DA SOCIEDADE BRASILERA DE ZOOTECnIA, 37., 2000, Viçosa-MG. Anais... Viçosa : Sociedade Brasileira de Zootecnia, 2000. CD-ROM.

MACEDO, W. Levantamento de reconhecimento dos solos do município de Bagé, RS. Bagé : EMBRAPA, 1984. 69p.

MELTON, A.A. et al. Milk production, composition and calf gains of Angus, Charolais and Hereford cows. Journal of Animal Science, v.26, n.4, p.804-809, 1967.

MORAES, J.C.; JAUME, C.M. A condição corporal como indicativo da atividade ovariana de vacas de corte criadas sob condições extensivas nas primeiras semanas pós-parto. Bagé : Cppsul, 2000. V.20, p.1-32. (Boletim de Pesquisa). 
NCSS 6.0. Statistical System for Windows - User's Guide I, II, III. Kaysville, Utah, 1996. 2204p.

NEVILLE, W.E. Jr. Influence of dam's milk production and other factors on 120 and 240-day weight of Hereford calves. Journal Animal Science, v.21, n.2, p.315-320, 1962.

OLIVEIRA, J.F.C. et al. Caracterização de aspectos produtivos de vacas Brangus Ibagé com distintos graus de fertilidade. Ciência Rural, Santa Maria, v.32, n.4, p.663-667, 2002.

PIMENTEL, C.A. et al. Effects of early weaning on reproductive efficiency in beef cows. Theriogenology, v.11, n.6, p.421-427, 1979.

PIMENTEL, M.A. et al. Produção de leite em bovinos de corte III. Peso ao parto e eficiência individual em novilhas Hereford e cruzas. In: REUNIÃO ANUAL DA SOCIEDADE BRASILERA DE ZOOTECNIA, 38., 2001, Piracicaba. Anais... Piracicaba :
Sociedade Brasileira de Zootecnia, 2001. p.393-394.

RUTLEDGE, J.J. et al. Milk yield and its influence on 205 - day weigth of beef calves. Journal Animal Science, v.33, n.3, p.563567, 1971.

SHORT, R.E. et al. Physiological mechanisms controlling anestrus and infertility in pospartum beef cattle. Journal of Animal Science, v.68, n.3, p.799-815, 1990.

SOUZA, C.J.H.; MORAES, J.C.F. Manual de sincronização de cio em ovinos e bovinos. Bagé : Embrapa Pecuária Sul, 1998. $76 p$.

SPITZER, J.C. et al. Reprodutive responses and calf birth and weaning weights as affected by body condition at parturition and pospartum weight gain in primiparous beef cows. Journal of Animal Science, v.73, p.1251-1257, 1995. 